\title{
Multilateral Study of the Andromeda Giant Stream: Implications for the progenitor, its MBH, and the M31 halo
}

\author{
Takanobu Kirihara ${ }^{1}$, Yohei Miki ${ }^{2}$, Masao Mori ${ }^{1,2}$, \\ Toshihiro Kawaguchi ${ }^{3}$ and R. Michael Rich ${ }^{4}$ \\ ${ }^{1}$ Faculty of Pure and Applied Physics, University of Tsukuba, Tennodai 1-1-1, Tsukuba, Japan \\ email: kirihara@ccs.tsukuba.ac.jp \\ ${ }^{2}$ Center for Computational Sciences, University of Tsukuba, Tennodai 1-1-1, Tsukuba, Japan \\ ${ }^{3}$ Sapporo Medical University, S1W17, Chuoh-ku, Sapporo, Japan \\ ${ }^{4}$ Department of Physics and Astronomy, University of California, Los Angeles, CA 90095, USA
}

\begin{abstract}
In a series of papers, we have performed $N$-body simulations of a galaxy collision to reproduce the observed shape, kinematics, and metallicity distribution of a giant stellar stream and shell-like structures in the halo of Andromeda galaxy (M31).
\end{abstract}

Keywords. galaxies: individual(M31)-galaxies: interactions-galaxies: kinematics and dynamics.

So far, a series of our studies have yielded constraints on the orbit, mass, and size of the progenitor using detailed comparisons between the results of the minor merger simulations and the observed data (Mori \& Rich 2008; Miki et al. 2014; Miki et al. 2016).

In Miki et al. (2014), we predicted that a hypothetical wandering supermassive black hole anchored in the progenitor nucleus lies within the halo (20-50 kpc away from the center of M31), and limits on its detectability with JVLA, ALMA, etc were calculated by Kawaguchi et al. (2014). Kirihara et al. (2014) investigated the density profile of the dark matter halo (DMH) in M31. To reproduce the giant stellar stream (GSS) and the stellar shells, the DMH density profile in the outskirts of M31 must be steeper than that of the prediction of the cold dark matter model.

Recently, we have paid attention to an asymmetric surface brightness profile of the GSS that decreases sharply on the north-eastern side and extends widely on the western side. We performed systematic parameter surveys of the merger simulation, varying the rotation velocity, thickness, and initial inclination of the progenitor disk galaxy. The result revealed that a non-rotating spherical progenitor fails to form the asymmetric surface brightness, and the progenitor's rotation is crucial in producing a realistic surface brightness profile (Kirihara et al. submitted). The model also successfully explains the observed metallicity gradient across the GSS and predicts another clear metallicity gradient in the western shell. Moreover, our high-resolution simulation predicts the existence of a faint stellar shell outside of the western shell that would be detectable by future observations.

\section{References}

Kawaguchi, T., Saito, Y., Miki, Y., \& Mori, M. 2014, ApJ, 789, L13

Kirihara, T., Miki, Y., \& Mori, M. 2014, PASJ, 66, L10

Kirihara, T., Miki, Y., Mori, M., \& Kawaguchi, T. 2016, submitted to MNRAS, arXiv e-prints (arXiv:1603.02682)

Miki, Y., Mori, M., Kawaguchi, T., \& Saito, Y. 2014, ApJ, 783, 87

Miki, Y., Mori, M., \& Rich, M. R. 2016, in press, arXiv e-prints (arXiv:1606.03173)

Mori, M. \& Rich, M. 2008, ApJ, 674, 77 